\title{
Structure and electronic properties of 4-6-12 graphene layers functionalized by fluorine
}

\author{
M.E. Belenkov ${ }^{\dagger}$, V.M. Chernov \\ †me.belenkov@gmail.com
}

Chelyabinsk State University, 129 Bratiev Kashirinykh St., Chelyabinsk, 454001, Russia

\begin{abstract}
This article presents the results of theoretical studies of new polymorphic varieties of fluorographene, which can be formed on the basis of $\mathrm{L}_{4-6-12}$ graphene layers. The calculations of the geometrically optimized structure, band structure, and density of electronic states were performed by the density functional theory method in the generalized gradient approximation. As a result of the theoretical analysis, it was established that three basic structural types of CF layers - T1, T2, and T3 - can be formed, which differ by the order of fluorine atom attachments. In these polymorphic varieties, all carbon atoms are in equivalent structural positions. When calculating the optimized structure of CF polymorphs, it was found that only T1 and T3 varieties have a stable structure. The structure of T2 type layers collapsed during the optimization. The crystal lattices of $\mathrm{CF}-\mathrm{L}_{4-6-12}-\mathrm{T} 1$ and $\mathrm{CF}-\mathrm{L}_{4-6-12}-\mathrm{T} 3$ are hexagonal, with 24 atoms in each of their unit cells. The layer density of the fluorographene layers is 1.42 and $1.52 \mathrm{mg} / \mathrm{m}^{2}$ for T1 and T2 types, respectively. The carbon-carbon bonds in the structure of the layers have different lengths and vary from 1.5157 to $1.6602 \AA$. The reason for this is the different number of electron pairs forming the corresponding covalent bond. The angles between bonds in CF layers vary over the wide range from $85.00^{\circ}$ to $133.47^{\circ}$, which is caused by deformation of the layer structure in comparison with the diamond structure. The sublimation energy of the T1 type fluorographene layer is $13.84 \mathrm{eV} /(\mathrm{CF})$, and the T3 layer is $13.80 \mathrm{eV} /(\mathrm{CF})$. Fluorographene polymorphs are semiconductors with the band gap of $3.193 \mathrm{eV}$ for the $\mathrm{CF}-\mathrm{L}_{4-6-12}-\mathrm{T} 1$ layer and $4.150 \mathrm{eV}$ for the $\mathrm{CF}-\mathrm{L}_{4-6-12}-\mathrm{T} 3$ layer.
\end{abstract}

Keywords: graphene, fluorographene, crystal structure, electronic properties, ab initio calculations.

УДК: 538.915

\section{Структура и электронные свойства 4-6-12 графеновых слоев функционализированных фтором}

\author{
Беленков М. Е. ${ }^{\dagger}$, Чернов В. М. \\ Челябинский государственный университет, ул. Братьев Кашириных, 129, Челябинск, 454001, Россия
}

В данной статье приведены результаты теоретических исследований новых полиморфных разновидностей фторографена, которые могут формироваться на основе $\mathrm{L}_{\text {4-6-12 }}$ графеновых слоев. Расчеты геометрически оптимизированной структуры, зонной структуры и плотности электронных состояний были выполнены методом теории функционала плотности в обобщенном градиентном приближении. В результате первичного теоретического анализа была установлена возможность формирования трех основных структурных типов СF-слоев - T1, T2 и Т3, которые отличаются порядком присоединения атомов фтора. В этих полиморфных разновидностях все атомы углерода находятся в эквивалентных структурных позициях. При расчетах оптимизированной структуры CF-полиморфов было установлено, что устойчивой структурой обладают только Т1 и Т3 разновидности. Структура слоев Т2 типа при оптимизации разрушилась. Кристаллические решетки $\mathrm{CF}-\mathrm{L}_{\text {4-6-12 }}-\mathrm{T} 1$ и $\mathrm{CF}-\mathrm{L}_{4-6-12}$-Т3 являются гексагональными, в их элементарных ячейках содержится по 24 атома. Слоевая плотность фторографеновых слоев составляет 1.42

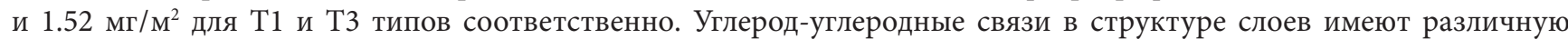
длину и варьируются от 1.5157 до $1.6602 \AA$. Причина этого различное число электронных пар, формирующих соответствующую ковалентную связь. Углы между связями в СF-слоях изменяются в широком диапазоне от $85.00^{\circ}$ до $133.47^{\circ}$, что вызвано деформацией структуры слоев по сравнению с алмазной структурой. Энергия сублимации фторографенового слоя Т1 типа составляет 13.84 эВ/(CF), а слоя Т3 - 13.80 эВ/(CF). Полиморфы фторографена являются полупроводниками с шириной запрещенной зоны, равной 3.193 эВ для слоя CF-L ${ }_{4-6-12}-\mathrm{T} 1$ и 4.150 эВ для слоя CF-L $\mathrm{L}_{4-6-12}-\mathrm{T} 3$.

Ключевые слова: графен, фторографен, кристаллическая структура, электронные свойства, первопринципные расчеты. 


\section{1. Введение}

Для широкого использования монослоев графена в наноэлектронных устройствах необходима модификация их электронных свойств, так чтобы из полуметаллических они стали полупроводниковыми $[1,2]$. Модифицировать свойства графеновых слоев возможно при механической деформации $[3,4]$ или за счет формирования полиморфных разновидностей графена, таких как $\mathrm{L}_{4-8}, \mathrm{~L}_{3-12}, \mathrm{~L}_{4-6-12}, \mathrm{~L}_{5-7}$ и других [5-8]. Кроме того, возможно изменить электронные свойства графеновых полиморфов в результате химической адсорбции на поверхности слоев различных неуглеродных атомов или молекулярных групп [9-13]. Из различных соединений на основе графена наиболее перспективным представляется фторографен, который обладает большей термической стабильностью по сравнению с графаном, хлорографеном и окисленным графеном. Структура фторографеновых слоев может быть различной в зависимости от порядка присоединения атомов фтора на поверхности слоя. Теоретические расчеты, выполненные ранее, показали, что возможно устойчивое существование 5 основных структурных типов при фторировании гексагонального $\mathrm{L}_{6}$ графена [14-16], 4 типов на основе слоя 4 - 8 [17] и одного типа на основе $\mathrm{L}_{3-12}$ графена [18]. Электронные свойства различных структурных типов фторографена изменяются в достаточно широких диапазонах, например, ширина запрещенной зоны может изменяться от 3.044 до 4.958 эВ [14-18]. В данной работе теоретически исследуется кристаллическая структура и электронные характеристики не изучавшихся ранее полиморфов фторографена, которые могут быть сформированы при фторировании $\mathrm{L}_{4-6-12}$ графена.

\section{2. Методы}

Теоретический анализ возможных способов присоединения атомов фтора к поверхности $\mathrm{L}_{\text {4-6-12 }}$ графена был выполнен в предположении, что число атомов фтора, присоединяющихся с каждой из сторон графенового слоя должно быть одинаковым. Рассматривались только основные полиморфные разновидности, в которых все позиции углеродных атомов являются структурно эквивалентными. Далее для модельно построенных периодических структур рассчитывалась геометрически оптимизированная структура, соответствующая такому пространственному расположению атомов, которое соответствует минимуму полной энергии $E_{\text {total }}$. Кроме того, выполнялись расчеты зонной структуры (BS) и плотностей электронных состояний (DOS). Все расчеты были выполнены методом теории функционала плотности (DFT) [19] в обобщенном градиентном приближении (GGA) [20] при помощи программного пакета Quantum ESPRESSO [21]. В расчетах использовались $12 \times 12 \times 12$ наборы k-точек и функционалы обменнокорреляционной энергии Педью-Зангера (PZ) [22], а также Педью-Берка-Ернзерхофа (РВE) [23]. Размерности набора базисных функций при вычислениях были ограничены значением энергии отсечки равным 70 Ридберг.

\section{3. Результаты}

Теоретический анализ возможных способов химической адсорбции атомов фтора на поверхности $\mathrm{L}_{4-6-12}$ графенового слоя показал, что возможно существование только трех основных структурных конфигураций, в которых позиции всех углеродных атомов в слое остаются структурно эквивалентными (Рис. 1). Если рассматривать соседние атомы углерода, к которым атомы фтора присоединяются с одной стороны слоя, как группу-кластер, то структурные типы слоев $\mathrm{L}_{4-6-12}$ фторированного графена отличаются числом атомов в этих кластерах. Для структурного типа Т1 в кластере один атом, а для типов Т2 и Т3 - два и шесть атомов соответственно (Рис. 1, Табл. 1).

При геометрической оптимизации методом DFT-GGA теоретически построенных соединений фторографена, построенных на основе $\mathrm{L}_{\text {4-6-12 }}$ графена, оказалось, что устойчивыми являются только СF слои типов Т1 и Т3. Структура фторографенового слоя Т2 типа разрушилась в процессе оптимизации. Изображения оптимизированной структуры CF-L ${ }_{4-6-12}-\mathrm{T} 1$ и $\mathrm{CF}-\mathrm{L}_{4-6-12}$-T3 слоев приведены на Рис. 2. Элементарные ячейки слоев относятся к гексагональным ячейкам Браве с точечной группой симметрии $6 \mathrm{~mm}$, в них содержится по 24 атома. Вектора элементарных трансляций слоев значимо отличаются - для СF слоя Т1 типа значение векторов $a=b=7.080 \AA$, а для слоя Т3 типа $-6.854 \AA$ (Табл. 1). Меньшее значение длин векторов для слоя Т3 обусловлено более сильной гофрированностью этого слоя по сравнению со слоем Т1. Отличие длин векторов трансляции структурных типов может быть использовано для их однозначной идентификацииметодамирентгеноструктурного анализа.

Расчетные значения слоевой плотности $\rho$ составляют

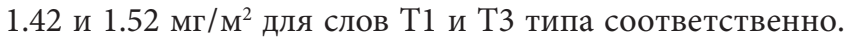
Слоевая плотность этих фторографеновых слоев выше плотности исходных слоев графена, но ниже плотности CF-L_-T1 слоя (Табл. 1). Значение длин векторов элементарных трансляций фторографеновых слоев на $2.0 \%$ или 5.4\% выше соответствующих значений исходных слоев графена $\mathrm{L}_{\text {4-6-12 }}$ (Табл. 1), что объясняется значительным увеличением длин межатомных углерод-углеродных связей при фторировании графенового слоя.

В Табл. 2 приведены значения расчетных длин межатомных связей и углов между ними для фторографеновых слоев. Каждый атом углерода в структуре слоев образует четыре ковалентные связи с соседними атомами. Связи были пронумерованы - фтор-углеродная межатомная связь была обозначена как первая (ее длина обозначена как $\mathrm{L}_{1}$ ). Длина этой связи варьируется от 1.4175 до $1.4350 \AA ̊$ (Табл. 2). Углерод-углеродная связь, обозначенная второй и имеющая длину $\mathrm{L}_{2}$, расположена между соседними четырех и шестиугольниками в структуре слоев $\mathrm{L}_{\text {4-6-12. }}$ Эта связь самая длинная по сравнению с остальными углерод-углеродными связями. Углерод-углеродные связи имеющие длины $\mathrm{L}_{3}$ и $\mathrm{L}_{4}$, располагаются между четырех- и двенадцатиугольниками, а также между шести- и двенадцатиугольниками в структурах CF-L $\mathrm{L}_{4-6-12}$ слоев соответственно (Рис. 2). 
Табл. 1. Структурные параметры и свойства полиморфных разновидностей Т1 и Т3 фторографена, сформированных на основе 4-6-12 графена, а также слоев $\mathrm{L}_{6}, \mathrm{~L}_{4-6-12}$ графена и CF- $\mathrm{L}_{6}$-T1 фторографена $(a, b-$ вектора элементарных трансляцй, $N-$ число атомов в элементарной ячейке, $\rho$ - плотность слоя, $E_{\text {total }}-$ полная энергия, $E_{\text {sub }}-$ энергия сублимации, $\Delta-$ ширина запрещенной зоны, $m$ - число атомов фтора в кластере, Def - деформационный параметр, * - единица измерения эВ/атом).

Table 1. Structural parameters and properties of polymorphic varieties of T1 and T3 fluorographene, formed on the basis of 4-6-12 graphene, as well as layers of $\mathrm{L}_{6}, \mathrm{~L}_{4-6-12}$ graphene and CF- $\mathrm{L}_{6}-\mathrm{T} 1$ fluorographene, $(a, b$ - elementary translation vectors, $N$ is the number of atoms in the unit cell, $\rho$ is the layer density, $E_{\text {total }}$ is the total energy, $E_{\text {sub }}$ is the sublimation energy, $\Delta$ is the band gap, $m$ is the number of fluorine atoms in the cluster, Def - deformation parameter, ${ }^{\star}$ - unit of measurement $\mathrm{eV} /$ atom).

\begin{tabular}{|c|c|c|c|c|c|}
\hline $\begin{array}{l}\text { Параметры } \\
\text { Parameters }\end{array}$ & CF- $\mathrm{L}_{4-6-12}-\mathrm{T} 1$ & $\mathrm{CF}-\mathrm{L}_{4-6-12}-\mathrm{T} 3$ & $\mathrm{CF}-\mathrm{L}_{6}-\mathrm{T} 1$ & $\mathrm{C}-\mathrm{L}_{6}$ & $\mathrm{C}-\mathrm{L}_{4-6-12}$ \\
\hline$a, \AA$ & \multirow{2}{*}{7.080} & \multirow{2}{*}{6.854} & \multirow{2}{*}{$2.602[15]$} & \multirow{2}{*}{$2.471[6]$} & \multirow{2}{*}{$6.713[6]$} \\
\hline$b, \AA$ & & & & & \\
\hline $\begin{array}{l}N \text {, атом } \\
N \text {, atom }\end{array}$ & 24 & 24 & 4 & 2 & 12 \\
\hline $\begin{array}{l}m, \text { атом } \\
m, \text { atom }\end{array}$ & 1 & 6 & 1 & - & - \\
\hline $\begin{array}{c}\rho, \mathrm{M \Gamma} / \mathrm{M}^{2} \\
\rho, \mathrm{mg} / \mathrm{m}^{2}\end{array}$ & 1.42 & 1.52 & 1.76 & 0.75 & 0.61 \\
\hline $\begin{array}{l}E_{\text {total }}, \ni \mathrm{B} /(\mathrm{CF}) \\
E_{\text {total }} \mathrm{eV} /(\mathrm{CF})\end{array}$ & -800.16 & -800.12 & $-800.64[15]$ & $-157.34^{*}[6]$ & $-156.65^{\star}[6]$ \\
\hline $\begin{array}{l}E_{\text {sub }}, \ni \mathrm{B} /(\mathrm{CF}) \\
E_{\text {sub }}, \mathrm{eV} /(\mathrm{CF}) \\
\end{array}$ & 13.84 & 13.80 & $14.32[15]$ & $7.78^{\star}[6]$ & $7.09^{\star}[6]$ \\
\hline $\begin{array}{l}\Delta, \text { эB } \\
\Delta, \mathrm{eV} \\
\end{array}$ & 3.193 & 4.150 & $3.321[15]$ & 0 & 0 \\
\hline Def, ${ }^{\circ}$ & 57.74 & 62.79 & 14.5 & 0 & 60 \\
\hline
\end{tabular}

Табл. 2. Длины межатомных связей (Li) и углы между связями ( $\left.\varphi_{i j}\right)$ в структуре полиморфных разновидностей Т1 и Т3 фторографена, сформированных на основе $\mathrm{L}_{4-6-12}$ графена.

Table 2. The lengths of interatomic bonds ( $\mathrm{Li})$ and the angles between bonds $\left(\varphi_{i j}\right)$ in the structure of polymorphic varieties T1 and T3 of fluorographene, formed on the basis of $\mathrm{L}_{4-6-12}$ graphene.

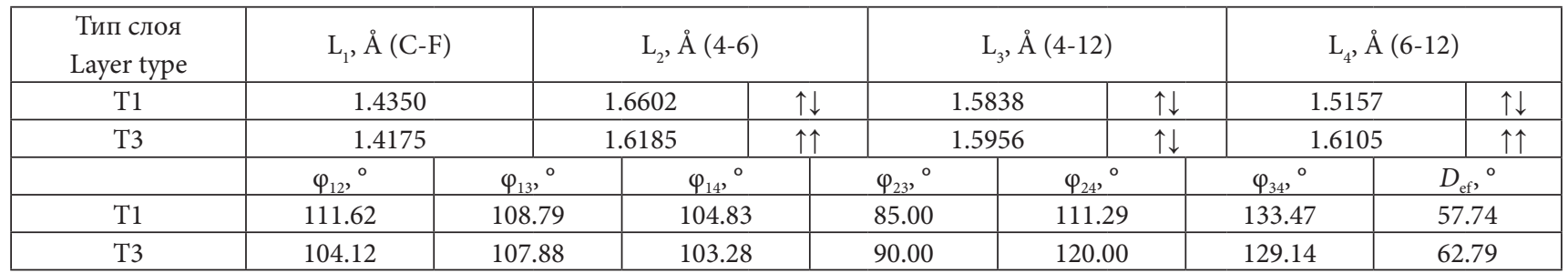

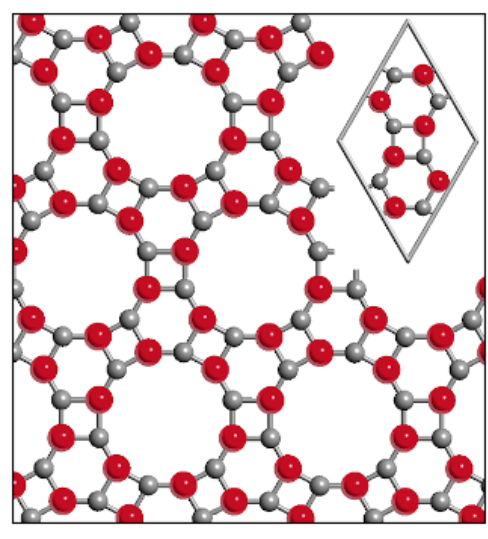

a

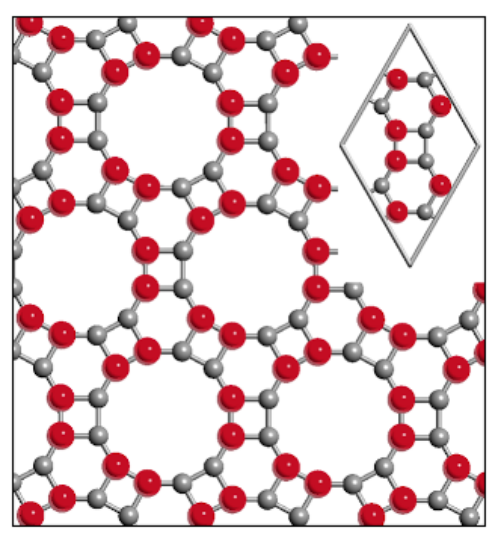

b

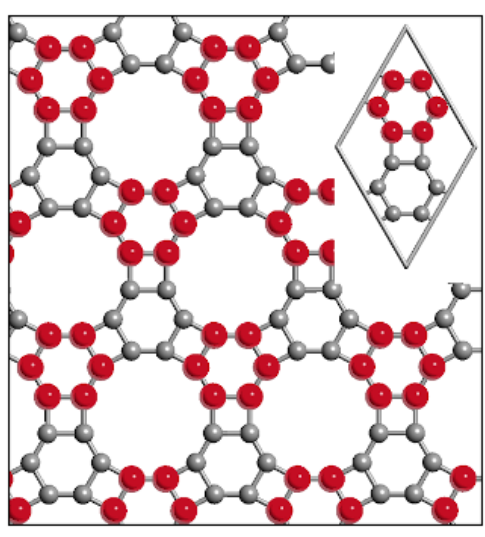

Puc. 1. (Color online) Возможные способы присоединения фтора к графеновому слою $\mathrm{L}_{4-6-12}$ : T1 - структурный тип 1 (а); T2 - структурный тип 2 (b); Т3 - структурный тип 3 (c). Разными цветами изображены углеродные атомы, к которым атомы фтора присоединяются с различных сторон графенового слоя. Все атомные позиции в слоях этих типов остаются эквивалентными.

Fig. 1. (Color online) Possible methods for attaching fluorine to the graphene layer $\mathrm{L}_{4-6-12}$ : T1 - structural type 1 (a); $2-$ structural type 2 (b); T3 is structural type 3 (c). Carbon atoms are depicted in different colors, based on the side of attachment to the graphene layer. All atomic positions in layers of these types remain equivalent. 

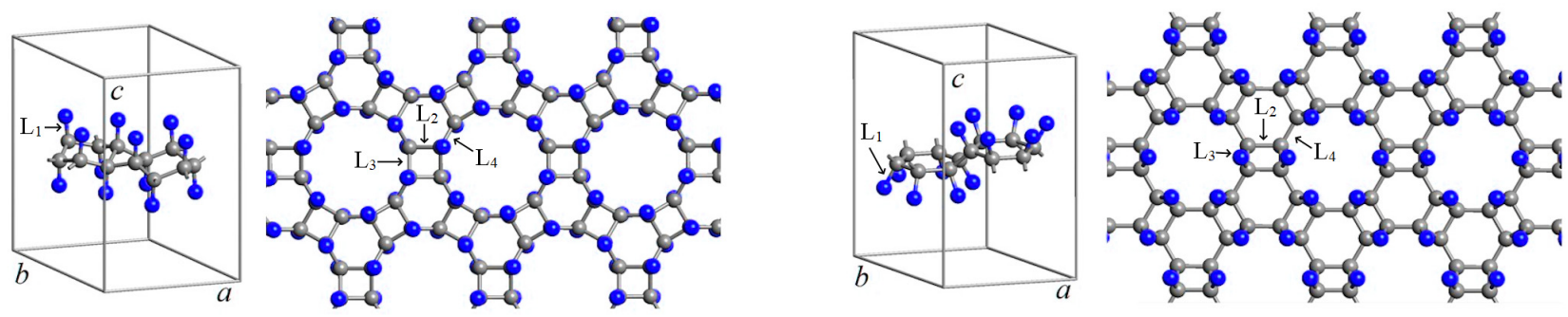

a

b

Pис. 2. (Color online) Элементарные ячейки и геометрически оптимизированная структура фторографеновых слоев, сформированных на основе графенового слоя $\mathrm{L}_{4-6-12}$ : Т1 - структурный тип 1 (a); Т3 - структурный тип 3 (b). Углеродные атомы и атомы фтора изображены серым и синим цветом соответственно.

Fig. 2. (Color online) Unit cells and the geometrically optimized structure of fluorographene layers formed on the basis of graphene layer $\mathrm{L}_{4-6-12}$ : T1 - structural type 1 (a); T3 is structural type 3 (b). Carbon and fluorine atoms are shown in gray and blue, respectively.

Различная длина связей между атомами углерода во фторографеновых слоях, по-видимому, обусловлена различным порядком этих связей, то есть различным числом электронных пар формирующих эти связи. Минимальный порядок связи соответствует максимальной длине $[24,25]$. Во фторографеновых слоях порядок связей минимален в многоугольных структурах с минимальным числом сторон. Также на длину углеродуглеродных связей, вероятно, должен влиять порядок присоединения атомов фтора к углеродным атомам атомы фтора могут присоединяться как с одной стороны слоя $\uparrow \uparrow$, так и с противоположных $\uparrow \downarrow$. Сравнительный анализ CF структур T1, Т2 и Т3 типов показал, что основным отличием структуры неустойчивого слоя Т2 типа от структур устойчивых слоев Т1 и Т3 заключается в том, что у третьей связи слоя Т2 ориентация присоединения атомов фтора сонаправленная, в то время как у устойчивых слоев она разнонаправленная (Табл. 2). Возможно, это является причиной неустойчивости слоя Т2 типа.

Значения углов между межатомными связями в слоях фторографена варьируются в интервале от $85.00^{\circ}$ до $133.47^{\circ}$ (Табл. 2). Так как углеродные атомы в СF слоях находятся в $s p^{3}$-гибридизированных состояниях, то оптимальное значение углов между связями должно составлять $109.47^{\circ}$, как наблюдается в структуре алмаза [24]. Отличие значений углов между связями от оптимальных можно рассматривать как деформацию их структуры по сравнению с идеальной. В качестве характеристики степени деформации можно использовать деформационный параметр Def, предложенный ранее для характеристики структуры алмазоподобных фаз [26]. Этот параметр был найден как сумма модулей разности углов между связями в слое и угла между связями в алмазе. Параметр деформации для слоя Т1 составляет $57.74^{\circ}$, а для слоя Т3 - 62.79. Значение параметров деформации фторографеновых слоев, формирующихся на основе графенового слоя $\mathrm{L}_{4-6-12}$ выше параметра $\mathrm{Def}=14.5^{\circ}$ для фторографенового слоя CF-L $-\mathrm{T} 1$ (Табл. 1). Различные значения деформационных параметров фторографеновых слоев, сформировавшихся на основе полиморфов графена $\mathrm{C}-\mathrm{L}_{6}$ и $\mathrm{C}-\mathrm{L}_{4-6-12}$, обусловлены различиями параметров Def исходных графеновых слоев (Табл. 1).
Энергии сублимации фторографеновых слоев CF-L ${ }_{4-6-12}$-T1 и CF-L ${ }_{4-6-12}$-Т3 составляют 13.84 и 13.80 эВ/(CF), соответственно (Табл. 1). Значения энергий сублимации хорошо коррелируют со значениями деформационных параметров - чем выше $E_{\text {sub }}$, тем меньше Def. Это общая закономерность, наблюдаемая как для фторографеновых слоев, так и для исходных слоев графена [6]. Энергии сублимации $\mathrm{CF}-\mathrm{L}_{4-6-12}$ слоев меньше $E_{\text {sub }}$ фторографеновых слоев, формирующихся на основе гексагонального графена $\mathrm{L}_{6}$ (Табл. 1). Однако значения энергий сублимации достаточно высокие для того чтобы фторографеновые слои на основе $\mathrm{L}_{4-6-12}$ графена могли устойчиво существовать при нормальных условиях.

Результаты DFT-GGA расчетов зонной структуры и плотности электронных состояний для фторографеновых слоев приведены на Рис. 3 и 4. Фторографеновые слои являются полупроводниками с шириной непрямой запрещенной зоны 3.193 и 4.150 эВ для слоев Т1 и Т3 типов, соответственно. Различная ширина запрещенной зоны у структурных типов обусловлена особенностями структуры, то есть порядком присоединения атомов фтора к слоям графена $\mathrm{L}_{4-6-12}$.

\section{4. Заключение}

Таким образом, в статье приведены результаты DFTGGA моделирования структуры и электронных свойств новых полиморфных разновидностей фторографена, которые могут образоваться при функционализации $\mathrm{L}_{4-6-12}$ графена фтором. В результате теоретического анализа установлена возможность существования трех основных структурных разновидностей $\mathrm{CF}-\mathrm{L}_{\text {4-6-12 }}$ слоев, из которых устойчивой структурой обладают только два слоя Т1 и Т3 типов. В элементарных ячейках CF слоев содержится по 24 атома, и они относятся к гексагональным ячейкам Браве с точной группой симметрии $6 \mathrm{~mm}$. Длины углерод-углеродных связей в слоях фторографена изменяются в диапазоне от 1.5157 до $1.6602 \AA$ А. Различия длин связи связано с различным порядком этих связей, то есть различным числом электронных пар формирующих эти связи - минимальный порядок связи соответствует её максимальной длине. Энергия сублимации фторографеновых слоев составляет порядка 13.8 эВ/(CF), что свидетельствует 

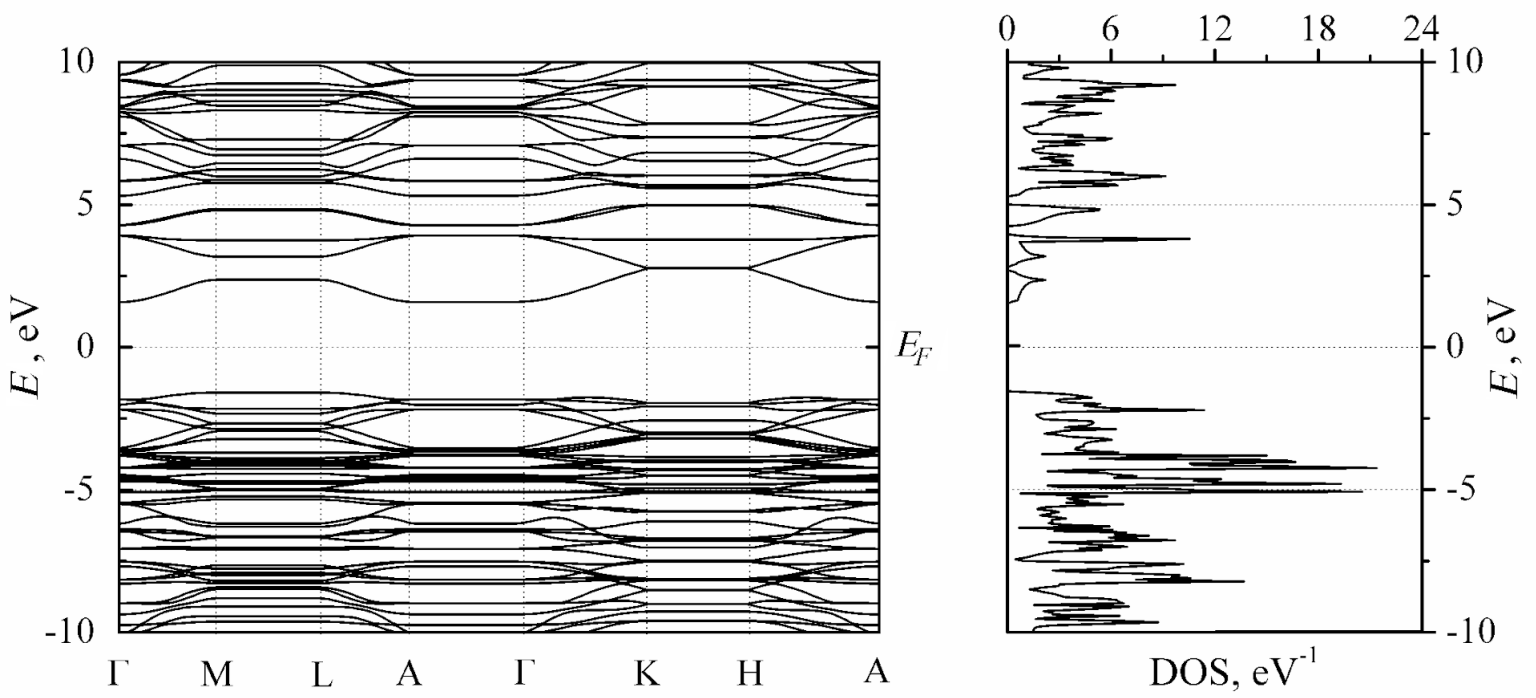

Рис. 3. Зонная структура и плотность электронных состояний Т1 полиморфной разновидности фторографена, сформированной при фторировании $\mathrm{L}_{4-6-12}$ графена.

Fig. 3. The band structure and the density of electronic states of the T1 polymorphic variety of fluorographene, formed during the fluorination of $\mathrm{L}_{4-6-12}$ graphene.
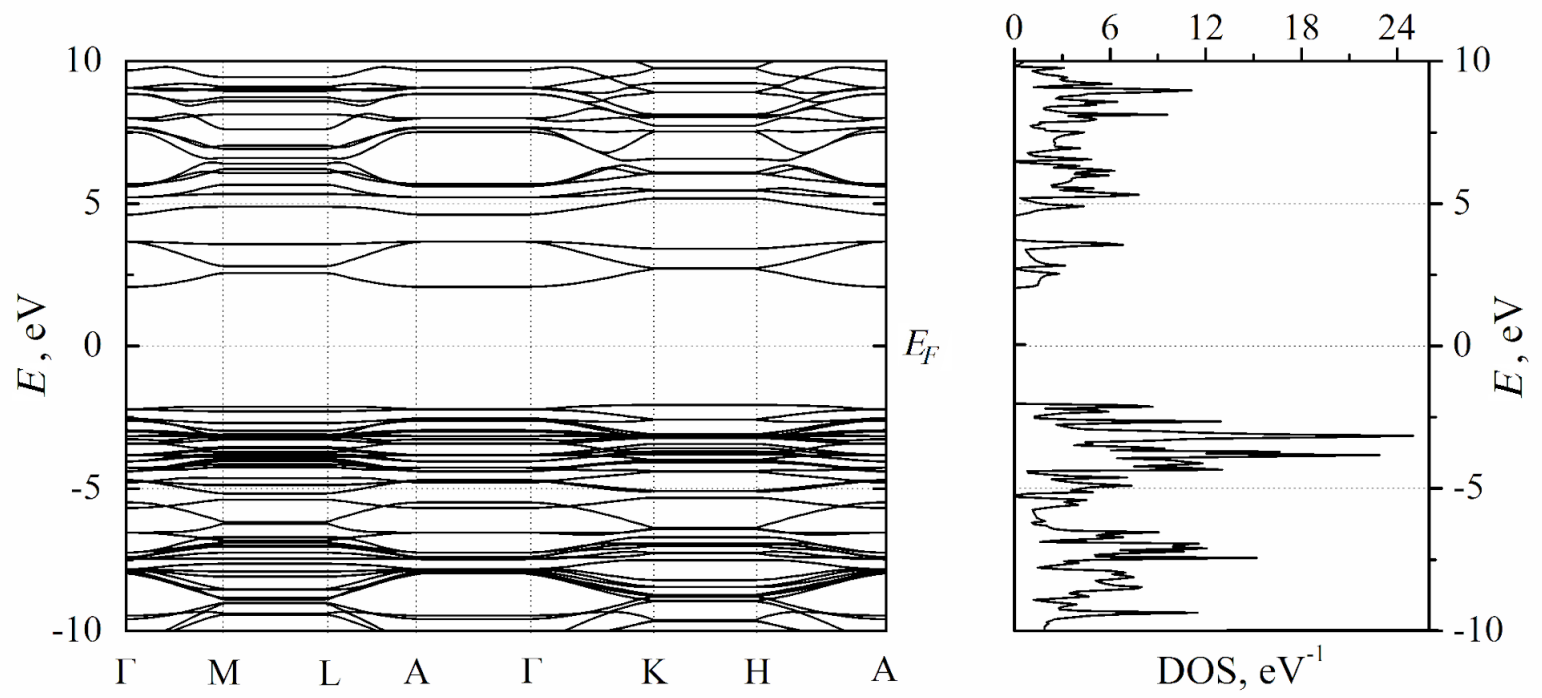

Рис. 4. Зонная структура и плотность электронных состояний Т3 полиморфной разновидности фторографена, сформированной при фторировании $\mathrm{L}_{4-6-12}$ графена.

Fig. 4. The band structure and the density of electronic states of the T3 polymorphic variety of fluorographene, formed during the fluorination of $\mathrm{L}_{4-6-12}$ graphene.

о возможности устойчивого существования этих соединений при нормальных условиях. Разница в энергиях сублимации полиморфных разновидностей Т1 и Т3 возможно связана с разницей энергии конфигураций взаимного расположения соседних атомов фтора как это было установлено ранее для графена, функционализированного водородом [27]. CF-L $\mathrm{L}_{\text {4-6-12 }}$ полиморфы фторографена являются полупроводниками с ширинами запрещенных зон изменяющихся от 3.193 до 4.150 эВ. Новые структурные типы фторографена, теоретически исследованные в данной статье, могут найти практическое применение при конструировании наноэлектронных устройств.
Благодарности / Acknowledgements. Исследование выполнено при финансовой поддержке РФФИ в рамках научного проекта № 20-32-90002. / The reported study was funded by RFBR according to the research project № 20-32-90002.

\section{Литература/References}

1. M. Freitag. Nature nanotechnology. 3 (8), 455 (2008). Crossref

2. G. Omar, M.A. Salim, B. R. Mizah, A. A. Kamarolzaman, R. Nadlene. In: Functionalized graphene nanocomposites and their derivatives. Ed. by M. Jawaid, R. Bouhfid, 
A.K. Qaiss. Amsterdam, Elsevier (2019) pp. 245-263. Crossref

3. S. V. Dmitriev, J. A. Baimova, A. V. Savin, Yu. S. Kivshar. Comput. Mater. Sci. 53, 194 (2012). Crossref

4. Yu. A. Baimova, S. V. Dmitriev, A. V. Savin, Yu. S. Kivshar. Phys. Solid State. 54 (4), 866 (2012). Crossref

5. A. Enyashin, A.L. Ivanovskii. Phys. Status Solidi B. 248 (8), 1879 (2011). Crossref

6. E.A. Belenkov, A.E. Kochengin. Phys. Solid State. 57 (10), 2126 (2015). Crossref

7. M.E. Belenkov, V.M. Chernov, E.A. Belenkov, V. M. Morilova. IOP Conference Series: Materials Science and Engineering. 447, 012005 (2018). Crossref

8. M.E. Belenkov, A.E. Kochengin, V.M. Chernov, E. A. Belenkov. IOP Journal of Physics: Conference Series. 1399, 022024 (2019). $\underline{\text { Crossref }}$

9. D. C. Elias, R. R. Nair, T. M. G. Mohiuddin, S. V. Morozov, P. Blake, M.P. Halsall, A.C. Ferrari, D. W. Boukhvalov, M.I. Katsnelson, A. K. Geim, K. S. Novoselov. Science. 323, 610 (2009). Crossref

10. J.T. Robinson, J.S. Burgess, C. E. Junkermeier, S.C. Badescu, T.L. Reinecke, F. K. Perkins, M. K. Zalalutdniov, J. W. Baldwin, J.C. Culbertson, P.E. Sheehan, E.S. Snow. Nano Letters. 10, 3001 (2010). Crossref

11. B. Li, L. Zhou, D. Wu, H. Peng, K. Yan, Y. Zhou, Z. Liu. ACS Nano. 5, 5957 (2011). Crossref

12. D. Chen, H. Feng, J. Li. Chem. Rev. 112, 6027(2012). Crossref

13. L.G. Bulusheva, A.V. Okotrub. In: New fluorinated carbons: fundamentals and applications. Amsterdam, Elsevier (2019) pp. $177-213$. Crossref

14. M.E. Belenkov, V.M. Chernov, E.A. Belenkov. Chelyabinsk Physical and Mathematical Journal. 3 (2), 202 (2018). (in Russian) [M.Е. Беленков, В. М. Чернов, Е. А. Беленков. Челябинский физико-математический журнал. 3 (2), 202 (2018).] Crossref
15. M.E. Belenkov, V.M. Chernov, E.A. Belenkov. IOP Journal of Physics: Conference Series. 1124, 022010 (2018). Crossref

16. K.S. Grishakov, K.P. Katin, M.M. Maslov, V.S. Prudkovskiy. Applied Surface Science. 463, 1051 (2019). Crossref

17. M.E. Belenkov, V.M. Chernov, E.A. Belenkov. IOP Conference Series: Materials Science and Engineering. 537, 022058 (2019). Crossref

18. M.E. Belenkov, V.M. Chernov. Physical and chemical aspects of the study of clusters, nanostructures and nanomaterials. 10, 406 (2019). (in Russian) [М.Е. Беленков, В.М. Чернов. Физико-химические аспекты изучения кластеров, наноструктур и наноматериалов. 10, 406 (2019).] Crossref

19. W. Koch, M. C. Holthausen. A chemist's guide to density functional theory. Weinheim, Wiley-VCH (2002) 313 p. Crossref

20. D.C. Langreth, M. J. Mehl. Physical Review B. 28 (4), 1809 (1983). Crossref

21. P. Giannozzi, S. Baroni, N. Bonini et al. J. Phys.: Condens. Matter. 21 (39), 395502 (2009). Crossref

22. J. P. Perdew, A. Zunger. Phys. Rev. B. 23 (10), 5048 (1981). Crossref

23. J. P. Perdew, K. Burke, M. Ernzerhof. Phys. Rev. Lett. 77 (18), 3865 (1996). Crossref

24. S. V. Shulepov. Fizika ugleroda. Chelyabinsk, Metallurgiya (1990) 336 p. (in Russian) [С. В. Шулепов. Физика углерода. Челябинск, Металлургия (1990) $336 \mathrm{c.}]$

25. E. A. Belenkov. Inorganic Materials. 37 (9), 928 (2001). Crossref

26. E.A. Belenkov, V.A. Greshnyakov. Physics of the Solid State. 58 (10), 2145 (2016). Crossref

27. K.P. Katin, V.S. Prudkovskiy, M.M. Maslov. Physics Letters A. 381, 2686 (2017). $\underline{\text { Crossref }}$ 